\title{
MENU DO DIA: SUSTENTABILIDADE - OS CONSUMIDORES ESTÃO DEGLUTINDO ESSA IDEIA?
}

\author{
Francisco Vicente Sales Melo \\ Doutorando em Administração pela Universidade Federal de Pernambuco - UFPE \\ Professora na Faculdade Ateneu - FATE \\ vicsmelo@gmail.com \\ Maria de Lourdes de Azevedo Barbosa \\ Doutora em Administração pela Universidade Federal de Pernambuco - UFPE \\ Professora na Universidade Federal de Pernambuco-UFPE \\ lourdesbarbosa@gmail.com \\ Salomão Alencar de Farias \\ Pós-doutor em Administração pela Georgia State University, EUA \\ Professora na Universidade Federal de Pernambuco - UFPE \\ saf@ufpe.br \\ Michelle Helena Kovacs \\ Doutora em Administração pela Universidade Federal de Pernambuco - UFPE \\ Professora na Universidade Federal de Pernambuco - UFPE \\ michellekovacs@gmail.com

\section{Sonia Rebouças da Silva Melo} \\ Doutora em Economia pela Universidade Federal de Pernambuco - UFPE \\ Professora na Universidade Federal de Pernambuco-UFPE \\ soniareboucas@gmail.com
}

\section{RESUMO}

A sustentabilidade é uma temática que ganhou importância no âmbito mercadológico e acadêmico. Assim, o objetivo deste artigo é examinar de que modo o argumento de sustentabilidade apresentado em um cardápio se relaciona com a avaliação mais positiva de um restaurante de hotel e a intenção de compra do consumidor. Para tanto, fez-se o uso de um quase-experimento. Os resultados indicam que o argumento de sustentabilidade disponível no cardápio produz avaliações mais positivas do restaurante por parte dos consumidores. Entretanto, os consumidores não estão propensos a frequentar e pagar mais por restaurantes que apresentam essas características em seu cardápio.

Palavras-chave: Cardápio; Consumidor; Restaurante; Sustentabilidade.

\section{DAY'S MENU: SUSTAINABILITY - ARE CONSUMERS SWALLOWING THIS IDEA?}

\begin{abstract}
Sustainability is a theme that has gained importance in marketing and academic circles. The aim of this paper is to examine how the sustainability argument, presented on a menu, is related to a more positive evaluation of a hotel's restaurant and to a consumer's purchase intent. The results of this quasi-experiment indicate that making the sustainability argument available on the menu leads to more positive assessments of the restaurant by consumers. However, they are not likely to attend and pay more for restaurants that have these features on their menu.
\end{abstract}

Key words: Consumer; Menu; Restaurant; Sustainability. 


\section{INTRODUÇÃO}

A discussão sobre sustentabilidade tem contribuído com a sensibilização dos indivíduos e gerado comportamentos sustentáveis por parte da sociedade desde a década de 1980. Em consequência disso, algumas pesquisas procuram verificar se essas questões realmente influenciam no comportamento dos indivíduos, tanto no que se refere à avaliação mais positiva de um produto ou serviço, quanto na decisão de compra.

Em 1990, nos Estados Unidos, devido ao aumento da demanda por uma alimentação saudável, a organização não-governamental (ONG) The Green Restaurant Association(2011) foi fundada com o objetivo de criar uma indústria de restaurantes ecologicamente sustentáveis. Com foco nesse aspecto da sustentabilidade, atualmente, a referida organização conta com mais de 300 restaurantes certificados ao redor do mundo, tendo desde pequenas cafeterias até grandes redes de serviços alimentícios. Para esta $\mathrm{ONG}$, há alguns requisitos que devem ser preenchidos para que um estabelecimento possa ser credenciado em sua rede. Os critérios vão desde a maneira de produzir os alimentos até a madeira escolhida para os móveis. Os serviços ofertados pela ONG estão relacionados com a avaliação ambiental, consultoria ambiental, certificação, marketing verde e relações públicas.

No Brasil, observa-se que há restaurantes que estão procurando se adequar às questões de sustentabilidade. Ainda que a culinária não seja especificamente orgânica, muitos priorizam uma comida mais brasileira, feita de alimentos produzidos regionalmente, procurando manter, por exemplo, os aspectos culturais. Em 2011, uma empresa brasileira que presta serviços de consultoria ambiental criou o selo 'Restaurante Sustentável', direcionado a restaurantes e bares que desejam comunicar suas ações de sustentabilidade no mercado. Para obtê-lo, os empreendimentos gastronômicos passam por um processo de certificação, que é necessário à adequação de diversos fatores pré-estabelecidos pela consultoria. Atualmente, sete estabelecimentos da cidade de São Paulo possuem este selo.

Sendo geralmente de iniciativa privada, os restaurantes são organizações que desempenham um papel importante no estilo de vida das pessoas. Comer fora é uma das atividades sociais que envolve o consumidor e tornou-se um hábito natural do cotidiano. Isso também ocorreu porque, nos últimos anos, a economia estável proporcionou maior incremento na remuneração, permitindo que os indivíduos possam optar por comer fora de suas residências (Walker; Lundberg, 2003). Por exemplo, segundo dados do Instituto Data Popular (2011), os brasileiros gastam hoje mais que o dobro do que gastavam há nove anos com alimentação fora de casa. Em 2002, essa despesa somava $\mathrm{R} \$ 59,1$ bilhões, enquanto no ano de 2011 os dados indicam que esses gastos subiram para $\mathrm{R} \$ 121,4$ bilhões.

Considerando a importância deste tema para este setor, provavelmente outras empresas deste ramo de atividade optem por uma gestão sustentável. Neste contexto, além de se adequarem a esse modelo, essas empresas também se preocupam em comunicar suas ações aos consumidores. Entre os vários itens relevantes de comunicação de um restaurante, têm-se, por exemplo, o cardápio. Gail (1993) afirma que este instrumento é um importante meio de comunicação entre o cliente e o restaurante, além de ser um item que pode refletir o estilo do local e, ao mesmo tempo, promover a cozinha do estabelecimento (Soriano, 2002; Strong, 2006). Com isso, é provável que práticas de sustentabilidade, divulgadas por meio deste canal, também possam influenciar nas atitudes dos consumidores, tanto no processo de avaliação e formação da imagem da empresa, quanto em suas intenções de compra (Biloslavo; Trnavcevic, 2009).

Deste modo, torna-se relevante investigar se um restaurante que apresenta características de sustentabilidade em seu cardápio pode se diferenciar dos demais e como estas características influenciam no processo de avaliação e intenção de compra. Assim, o objetivo central deste artigo é examinar de que modo o argumento de sustentabilidade apresentado em um cardápio se relaciona com a avaliação mais positiva de um restaurante de hotel e a intenção de compra do consumidor. Tendo contextualizado o problema de pesquisa, apresenta-se, em seguida, a revisão da literatura sobre a temática e o método de investigação utilizado. Posteriormente, são descritas as análises e 
discussões, fechando com as conclusões e as referências utilizadas para a articulação teórica deste estudo.

\section{REVISÃO BIBLIOGRÁFICA}

Estudos afirmam que as pessoas escolhem um restaurante para comer tanto por necessidades relacionadas ao prazer e diversão, quanto por significados práticos e simbólicos; isso diferentemente para cada uma dessas situações ou estilo de pessoa. Por exemplo, Kivela (1997) considera que o momento no qual as pessoas pretendem vivenciar uma ocasião, seja social, de conveniência, celebração ou negócios, é fator determinante para a escolha de um restaurante. Neste estudo, a intenção é verificar se a questão da sustentabilidade também se apresenta como um fator relevante para o consumidor, não apenas no seu processo de escolha, mas também no de avaliação do estabelecimento e intenção de compra.

\subsection{Restaurantes e a escolha do consumidor}

Lewis (1981) considera que há cinco atributos importantes para a escolha de um restaurante: a qualidade da comida, a variedade do cardápio, o preço, a atmosfera e os fatores de conveniência. A qualidade da comida foi avaliada como a variável que mais influenciava no retorno e aumento da frequência em um restaurante, todavia a importância dada aos outros atributos sofria variações de acordo com o tipo de restaurante. Por exemplo, a atmosfera foi considerada mais relevante na escolha de restaurantes do tipo gourmet e temáticos do que na escolha dos mais familiares e populares. Esses achados alinham-se aos de Dorf (1992), quando afirma que o design de um restaurante deve estar cuidadosamente alinhado com sua localização, cardápio, preço, imagem no geral e, principalmente, com seu estilo para que possa atingir seu público-alvo.

Ao analisarem o consumo em um restaurante como uma experiência de aspecto multidimensional, Andersson \& Mossberg (2004) concluíram que os clientes estão dispostos a gastar mais com os seguintes itens: interior dos restaurantes, a qualidade dos serviços prestados, companhia e a presença de outros clientes que preenchem suas necessidades sociais e intelectuais, durante o jantar. Cabe ressaltar que os estudos sobre os restaurantes, geralmente, destacam que essas empresas caracterizam-se por serem organizações prestadoras de serviços complexos que exigem contato entre os profissionais e os consumidores na entrega do serviço, além de proverem tanto produtos físicos quanto serviços (Kotler, 1973). Entretanto, estudos mostram que é necessário não só criar percepções para os aspectos tangíveis do serviço, mas também proporcionar evidências sólidas da experiência intangível, composta por elementos abstratos (por exemplo, interação com os funcionários) do que se quer vender (Barbosa; Kovacs, 2004).

Pesquisas mais recentes (Baker; Parasuraman; Grewal; Voss, 2002; Lin 2004) mostram que pessoas respondem ao cenário de serviços de maneira diferenciada. Mesmo que percebam estímulos independentes, é a composição total de estímulos que determina a resposta dos indivíduos. Com isso, cada dimensão pode afetar a configuração total, independentemente ou por meio de suas interações com as demais dimensões. Para Bitner (1992), é necessário variar diversos elementos do servicescape simultaneamente para atingir uma percepção do todo que possa influenciar de forma significativa um comportamento.

Embora existam várias evidências de que o conjunto, ou seja, a atmosfera do ambiente, influencia mais nas atitudes do consumidor nos restaurantes do que aspectos individuais, aqui a preocupação é apenas com um dos aspectos tangíveis que compõe o ambiente de um restaurante, no caso, o cardápio composto de argumentos de sustentabilidade.

O termo cardápio é um neologismo criado em 1899 pelo filósofo brasileiro, Antônio de Castro, para substituir o termo francês menu (Houaiss; Villar, 2004). Esta palavra, em seu sentido original, significa carta, ementa, lista, precário, equivalendo-se ao português de Portugal que significa ementa. Com relação ao termo menu, esses autores definem como a lista detalhada de carnes que compõe uma refeição ou lista de refeições. Segundo Castelli (1999), a origem do termo 
adotado na língua portuguesa brasileira explica, em partes, diferentes terminologias utilizadas para se referir ao cardápio, variando em carta de comidas e carta de menu. As discussões existentes quanto à semântica contribuem para a fixação do termo, além de auxiliar no processo de sua definição. No entanto, verifica-se que o debate existente tende a ser insignificante, já que há diferenças pequenas entre os pontos de vista apresentados pelos autores. Portanto, admite-se, neste estudo, que cardápio e menu são sinônimos.

$\mathrm{Na}$ perspectiva teórica, o principal caminho para que os consumidores dimensionem a qualidade da comida é por meio do design do cardápio (Soriano, 2002). Este instrumento é considerado uma ferramenta de vendas bastante relevante para o setor de restaurantes. Gail (1993) considera também que o menu é um importante meio de comunicação entre o cliente e o restaurante, devendo refletir a característica do local, ser informativo de maneira que promova a cozinha do estabelecimento, passando estilo, integridade e segurança ao consumidor. Corroborando com esta ideia, Strong (2006) destaca que o cardápio trata-se da declaração da filosofia do restaurante, uma crucial parcela da compreensão do marketing, e deve capturar a forma, o design, a função, marca e o estilo.

Segundo Pelaez (2008), no processo de planejamento de um cardápio, o gestor do estabelecimento deve está atento às novidades e às tendências de mercado, num processo contínuo de profissionalismo, qualidade e responsabilidade. A autora considera que este é um consenso existente entre os pesquisadores que estudam o assunto e, além disso, os menus devem ser influenciados pelas novas tendências da alimentação quanto ao produto ofertado, tipo de alimentos, métodos de cocção, qualidade dos ingredientes, formato e apresentação.

Quanto aos aspectos relacionados à forma, cresce a preferência por cardápios curtos, com poucas opções (Maricato, 2005). Segundo Walker \& Lundberg (2003, p. 183), o "[...]design e o layout do cardápio são denominados os vendedores silenciosos de um restaurante". Para os autores, o design do menu refletirá o ambiente do restaurante e as imagens e textos que o compõem tendem a passar para o consumidor a essência do local. É por isso que para a elaboração deste instrumento, é necessário obter ajuda de designers ou técnicos em computação gráfica para que os cardápios sejam elaborados em sintonia com a decoração, o ambiente e os aspectos relacionados à identidade da empresa.

Por outro lado, com relação à elaboração do cardápio no contexto dos restaurantes, alguns autores consideram que geralmente devem ser elaborados com base em questões quantitativas, tais como preço, custo e itens de produtividade (Bayou; Bennett, 1992). Todavia, Jones e Mifli (2000) sugerem que, na elaboração, o gestor trabalhe com abordagens quantitativas e qualitativas. Nesta primeira, os autores apontam que se deve considerar o volume da popularidade dos produtos, a lucratividade e a margem de contribuição. Já nos aspectos qualitativos, a qualidade, o sabor e a apresentação do alimento, bem como outras variáveis que possam informar ações desenvolvidas pelo restaurante. Nesta abordagem, é possível analisar com mais profundidade as características relevantes para o processo de planejamento dos cardápios, sendo possível incorporar elementos tangíveis e intangíveis, que venham facilitar o processo de decisão de compra por parte do consumidor.

Para que um ato de compra se concretize, os consumidores, de maneira geral, realizam avaliações e julgamentos entre produtos e marcas ofertados. Esse processo de avaliação trata-se do julgamento, conhecido como pistas associadas aos produtos ou serviços. As pistas seriam entendidas, então, como os vários itens de informações sugestivas sobre os produtos, as marcas ou os serviços, que, uma vez identificados, ajudam a compor uma avaliação (Szybilo; Jacoby, 1974). Deste modo, com relação à avaliação de um restaurante de hotel por meio do seu menu, é provável que os indivíduos que pretendem consumir os produtos ofertados, tenham atitudes positivas com relação a características de sustentabilidade disponíveis em seu cardápio, item apresentado a seguir. 


\subsection{O consumidor frente às questões de sustentabilidade}

Devido às várias discussões sobre a sustentabilidade, com destaque para o tema meio ambiente, a consciência ambiental tem germinado na sociedade. Em consequência disso, os consumidores têm sido influenciados por estas questões em seus comportamentos de compra. Vários estudos analisam o consumidor na compra de produtos ecologicamente corretos e seus interesses ambientais e de sustentabilidade (Herberger, 1975; Tucker, 1980; Martin; Simintiras, 1995; Lee; Holden, 1999; Chen; Chai, 2010; Okada; Mais, 2010), seu perfil, considerando as variáveis demográficas e psicográficas, (Kassarjian, 1971; Anderson; Cunningham, 1972; Kinnear et al., 1974; Brooker, 1976; Murphy et al., 1978; Leonard-Barton, 1981; Aaker; Bogazzi, 1982; Hume et al., 1989; Samdahl; Robertson, 1989; Zimmer et al., 1994; Roberts, 1996; Roberts; Bacon, 1997; Smith, 2001; Laroche; Bergeron; Forleo, 2001; Rowlands, Scott, Parker, 2003; Firat, 2009; Awad, 2011) e suas ações e atitudes de consumo consciente (Lee; Holden, 1999; Okada; Mais, 2010; Robinot; Giannelloni, 2010), tendo sido um tema importante dentro do campo.

Embora identificados poucos estudos que tratam desses aspectos comportamentais com relação a restaurantes ou algum tipo de serviço relacionado, observa-se que aqueles que analisam as atitudes com relação a outros aspectos como embalagens (Lages; Neto, 2002; Pereira; Ayrosa, 2004), sinalizam de que modo os consumidores vêm se comportando quanto às questões de sustentabilidade, principalmente, no que se refere ao meio ambiente. Por exemplo, Manaktola \& Jauhari (2007), exploraram as atitudes dos consumidores com relação a práticas verdes no setor hoteleiro na Índia. Os autores demonstraram que os consumidores avaliam positivamente e estão mais propensos a consumir produtos e serviços que desenvolvam ações ambientais e sustentáveis, mas não estão dispostos a pagar mais por estes serviços. Destacam que fazem escolhas com base na combinação de atributos que melhor atenda suas necessidades. Além disso, consideram que atributos que são diferentes dos básicos podem gerar valor agregado ao negócio.

Com o objetivo de examinar se ações 'verdes' praticadas por hotéis contribuem para a satisfação dos clientes, Robinot \& Giannelloni (2010) realizaram um estudo qualitativo que ajudou a gerar uma lista de características que contribuem para a satisfação geral com um hotel. Os principais resultados mostraram que os atributos ambientais foram avaliados como básicos; o que significa que foram vistos como parte integrante da oferta de serviços e não como critérios diferenciadores. Os pesquisadores sugeriram então que é necessário manter um alto nível de ações ambientalmente corretas e as empresas devem adotar práticas sustentáveis, mesmo que não influenciem na satisfação dos consumidores.

Por outro lado, numa perspectiva mais abrangente, Okada \& Mais (2010) defendem que os indivíduos estão mais sensíveis às questões ambientais e sustentáveis e concordam com a ideia de que os consumidores estão dispostos a pagar um prêmio, ou seja, um valor maior por produtos e serviços 'verdes'. O objetivo do trabalho destes autores foi verificar como os consumidores respondem às alternativas ecológicas e examinar como os gestores das empresas podem melhor posicionar seus produtos verdes com preços que os consumidores estejam dispostos a pagar. Os resultados indicaram que o enquadramento positivo (enfocando as vantagens do produto verde) funciona melhor para os consumidores ambientalmente conscientes, enquanto o enquadramento negativo (com foco em evitar as desvantagens do produto não-verde) funciona melhor para aqueles menos conscientes. Além disso, observaram que os consumidores estão dispostos a pagar mais pelas alternativas ambientalmente corretas.

Com relação ao produto em si, Martin \& Simintiras (1995) realizaram um estudo sobre as atitudes em relação aos produtos verdes e seu impacto sobre o meio ambiente. Os resultados sugerem que não existe nenhuma relação direta entre os produtos ecologicamente corretos, conhecimentos específicos sobre o produto e as atitudes ambientais, e que os consumidores simplesmente não acreditam que um produto verde seja bom para o ambiente, principalmente quando não sabem quais são os impactos que esse produto provoca no meio ambiente. Ou seja, os 
indivíduos estão mais propensos ao consumo ambientalmente correto quando sabem os benefícios de suas ações.

Para Chen \& Chai (2010), o crescimento rápido da economia e os padrões mundiais de consumo são as principais causas da deterioração ambiental. Consideram que os problemas ambientais continuam a se agravar e que, por ser considerada uma grande preocupação pública, os países devem aderir ao movimento verde. Os autores fizeram um estudo com o objetivo de comparar o gênero das pessoas com atitudes em relação ao meio ambiente e produtos verdes, analisando também a relação entre atitudes, meio ambiente e produtos verdes. Os resultados iniciais mostraram que não há diferenças significativas entre o gênero e as atitudes ecológicas dos consumidores. Viram que o papel desempenhado tanto pelo governo quanto pelas normas de sustentabilidade contribuem significativamente para uma atitude mais positiva em relação à esse tipo de produto.

Considerando então que as pesquisas com relação às atitudes dos consumidores em relação às questões de sustentabilidade e ecológicas ainda estão em evolução, e que os estudos mais recentes vêm tentando teorizar e testar empiricamente se os consumidores apresentam atitudes avaliativas mais positivas e intenções de compra com relação a estas, formulou-se a seguinte hipótese de pesquisa: Hipótese 1: Os consumidores terão atitudes mais positivas na avaliação de restaurantes que enfatizam práticas de sustentabilidade em seu cardápio.

Informações relativas aos produtos, consideradas como as pistas disponíveis (Szybilo; Jacoby, 1974), devem, a princípio, gerar respostas (atitudes e comportamentos) dos consumidores. Tomando por base a ideia presente na literatura de que argumentos de sustentabilidade e ecológicos levam a atitudes favoráveis e aumentam a preferência do consumidor (Pereira; Ayrosa, 2004; Ottman, 1994), considera-se que um argumento de sustentabilidade, veiculado por meio de um instrumento de estímulo mercadológico, no caso o cardápio de um restaurante, pode influenciar na decisão de compra por parte do consumidor. Deste modo, formulou-se a seguinte hipótese de pesquisa: Hipótese 2: Os consumidores, em geral, estão propensos a frequentar e pagar mais por restaurantes que apresentam características de práticas sustentáveis em seu cardápio.

\section{PROCEDIMENTOS METODOLÓGICOS}

Este estudo é de natureza explicativa; Winer, 1999; Churchill, 1999; Aaker; Kumar; Day, 2001; Malhotra, 2006; Corrar; Paulo; Dias Filho, 2007; Lattin; Carroll; Green, 2011) e se enquadra na modalidade de quase-experimento (Churchill, 1999; Grimshaw et al., 2000; Shadish; Cook; Campbell, 2002; Malhotra, 2006).

Apesar das críticas, como a não possibilidade de se configurar um laboratório sem interferências de variáveis estranhas e de que os participantes não estarão vivenciando algo real no momento da pesquisa, o quase-experimento é aceito na academia e usado em áreas como administração e psicologia (por exemplo, Cohen; Ledford, 1994; Mayer; Davis, 1999; Holder; Berndt, 2011; Grant; Hofmann, 2011), e vem sendo utilizado também para pesquisas em marketing (Szulanski; Jensen, 2004; Smith, 2006; Debruyne; Frambach; Moenaert, 2010). Verificou-se também que é mais comum a utilização de modelos quase-experimentais em estudos que procuram analisar, por exemplo, atitudes e argumentos ecológicos com relação a produtos (Serpa; Alves, 2000; Pereira; Ayrosa, 2004; Velter, et al., 2009), sendo esta uma oportunidade para adequar e ampliar as análises para o campo dos serviços de restauração.

O conjunto universo considerado para esta pesquisa compreende todos os possíveis consumidores de serviços alimentícios ofertados por restaurantes, aqui considerados como infinito. Devido ao caráter não-probabilístico aqui adotado, o critério de definição do número de respondentes foi baseado nas normas práticas (Hair et al., 2006) e na observação das médias de tamanho de amostra observados em estudos anteriores. Neste sentido, com base em alguns estudos de campo na área do marketing (Serpa; Avila, 2000; Petroll; Damacena; Zanluchi, 2004; Pereira; 
Ayrosa, 2004), foi definido que deveriam ser entrevistados, no mínimo, 60 respondentes por grupo, para que, após o tratamento dos formulários, restasse no total uma quantidade mínima de 100 questionários. De acordo com Hair, et al. (2006), Malhotra (2006) e Lattin, Carroll \& Green (2011), para o uso das análises variadas e multivariadas, é necessária uma amostra mínima de 50 respondentes. Seguindo esta orientação, procedeu-se a coleta de dados. Ao final do tratamento e da validação, obteve-se 113 questionários válidos, sendo 58, do grupo de controle; e 55, do grupo de experimento.

\subsection{Cenário de pesquisa}

Dois cenários foram criados: um para o grupo de controle $(\mathrm{GC})$ e outro para o grupo de experimento (GE) (Hulland; Chow; Lam, 1996). O primeiro observou a imagem de um cardápio de hotel com ofertas semanais em seu formato original. O segundo, por sua vez, observou o cardápio com elementos adicionais, ou seja, esse grupo passou por uma situação induzida de informação voltada à sustentabilidade (selo), por meio da manipulação das informações apresentadas neste menu. A escolha do cardápio como cenário se deu devido a sua importância no processo de comunicação de serviços prestados por restaurantes (Bayou; Bennett, 1992; Gail, 1993; Walker; Lundberg, 2003).

$\mathrm{O}$ cardápio usado foi retirado da internet, eleito de forma aleatória. Na pesquisa, deu-se preferência por modelos simples e que apresentassem refeições completas para facilitar a apreciação dos participantes. O argumento de sustentabilidade usado foi o selo de 'Restaurante Sustentável', criado por uma empresa de consultoria de serviços ambientais em São Paulo. O selo foi colocado no canto inferior direito com a ajuda de um software de manipulação gráfica. Depois de examinar as figuras, os participantes preencheram um questionário com as medidas das variáveis dependentes e independentes.

A pesquisa foi conduzida em locais fechados, climatizados e preparados exclusivamente para o processo de investigação. A sala está localizada em uma instituição do ensino superior situada na região nordeste do Brasil, sendo esta preparada para ser o laboratório de pesquisa. Ressalta-se que estudos desta natureza possibilitam melhor controle sobre as variáveis independentes pertinentes ao problema de pesquisa, resultando maior rigorosidade no processo (Hulland; Chow; Lam, 1996; Sampieri, et al., 2006).

\subsection{Variáveis, instrumento e coleta de dados}

Na revisão da literatura, não foi localizado nenhum instrumento específico que mensure aspectos de avaliação e intenção de compra para restaurantes. Com base no que foi levantado, verificou-se que, para atender aos objetivos propostos, seria necessário trabalhar com quatro dimensões: design do cardápio, avaliação do restaurante, sustentabilidade e intenção comportamental.

Para a dimensão design do cardápio, consideraram-se as contribuições teóricas existentes na literatura. Os autores Gail (1993), Soriano (2002) e Strong (2006), afirmam que os consumidores dimensionam a qualidade da comida por meio do design do cardápio. Além desses, Walker \& Lundberg (2003) asseguram que o design e o layout do cardápio são denominados os vendedores silenciosos de um restaurante. Quanto à dimensão avaliação do restaurante, considera-se a ideia das pistas necessárias para compor este processo e formação da imagem por parte do consumidor (Szybilo; Jacoby, 1974).

Com relação à sustentabilidade, consideraram-se as perspectivas levantadas na literatura e resultadas de estudos relacionados às questões ambientais e de sustentabilidade (Herberger, 1975; Tucker, 1980; Martin; Simintiras, 1995; Lee; Holden, 1999; Chen; Chai, 2010; Okada; Mais, 2010). Quanto às intenções de compra, foi realizada uma adaptação da escala Purchase Intentions Scale, (PI) proposta por Schwepker \& Cornwell (1991). A concordância do respondente será indicada por 
meio de escala tipo Likert (Malhotra, 2006). O ponto 1 representará discordo totalmente e o 5, concordo totalmente; o ponto médio 3 é considerado como nem concordo nem discordo.

Os dados foram coletados por meio de um questionário estruturado e fechado. Além das escalas para medição das variáveis dependentes, o instrumento foi composto por seis questões fechadas para levantamento do perfil demográfico dos grupos e outras quatro questões relacionadas com as práticas e preferências com relação a restaurantes (Churchill, 1999; Grimshaw et al., 2000; Shadish; Cook; Campbell, 2002; Sampieri et al., 2006; Malhotra, 2006).

Conforme orientação de Malhotra (2006), os questionários foram todos numerados e todas as opções de respostas, codificadas. O pré-teste do instrumento, realizado juntamente com o préteste do cenário, apresentou resultados favoráveis para realização da pesquisa. Ressalta-se que todas as seções de experimento foram aplicadas pelo pesquisador, ou seja, não houve auxílio externo para coleta de dados.

A operacionalização da pesquisa se deu da seguinte forma (Cooper; Shindler, 2003; Sampieri et al., 2006; Malhotra, 2006): a) Os respondentes foram divididos em grupos por conveniência. Em seguida, foi realizado um sorteio aleatório para definir quais grupos são de controle ou experimento; b) Após a definição dos grupos, os participantes foram informados que se tratava de uma pesquisa de natureza acadêmica; c) $\mathrm{O}$ objetivo de analisar se os aspectos de sustentabilidade influenciam na avaliação mais positiva e intenção de compra em restaurantes não foi revelada como forma de não influenciar os respondentes; d) Os dois cenários foram apresentados por meio de um projetor de imagens. Feito isso, foi pedido que os participantes imaginassem que estavam em uma situação de compra num restaurante de hotel. Foi dado um tempo de cinco minutos antes da entrega dos questionários - cada participante respondeu um ; e) Embora o questionário seja autoaplicável, foi dada uma breve explanação, como forma de minimizar erros de amostragem; e f) Ao final, as imagens foram retiradas e os questionários, recolhidos.

Os procedimentos adotados para preparar a escala foram os testes de aderência e confiabilidade (Aaker; Kumar; Day, 2001; Malhotra, 2006). Realizou-se também testes de normalidade, cálculo do coeficiente alfa de Cronbach e testes de médias. As análises foram finalizadas com estatísticas descritivas, multivariadas e os testes de hipóteses. Todo esse processo foi realizado com o auxílio de um pacote estatístico bastante utilizado nas ciências sociais. Para a avaliação da validade, foi realizado um confronto teórico (articulação teórica com os achados), buscando identificar uma validade convergente ou divergente do que outros resultados indicam (Cooper; Schindler, 2003).

\section{RESULTADOS E ANÁLISES}

Participaram da pesquisa 60 respondentes, sendo obtidos 58 questionários válidos no grupo de experimento, contra 57 entrevistados e 55 questionários válidos no grupo de controle. $\mathrm{Na}$ amostra experimental, houve proporção semelhante de homens (52\%) e mulheres (48\%), enquanto no grupo de controle, o percentual de homens (64\%) foi mais elevado. Nos dois grupos, predominaram os jovens com idades na média de 22 (GE) e 24 (GC) anos, solteiros, residentes no Estado de Pernambuco, distribuídos em 21 municípios do Estado. Todos são, atualmente, estudantes do ensino superior dos cursos de administração e economia, com renda familiar variando em torno de R\$3.287,00 (GE) e R \$ 2.942,00 (GC).

A maioria dos participantes frequenta $(78 \%)$ restaurante mensalmente. Cabe ressaltar que o GC apresenta maior percentual de frequentadores (87\%). Além disso, observou-se que, também em sua maioria $(79,6 \%)$, os respondentes afirmaram que praticavam ações ambiental e social, sendo $84,5 \%$, no grupo experimental e 74,5\%, no grupo de controle. Para ambos, considerando aqueles que costumam ir a restaurantes, os homens $(60,2 \%)$ tendem a frequentar mais que as mulheres 
$(39,8 \%)$. Por outro lado, observa-se um equilíbrio nas respostas sobre atitudes ambientais e sociais para os dois gêneros (homens e mulheres), o que corrobora com os achados de Chen e Chai (2010).

Para testar a aderência da amostra, utilizou-se o teste de Kolmogorov-Smirnov (K-S), conforme sugere Malhotra (2006). O teste K-S compara a função de distribuição cumulativa de uma variável com uma distribuição específica. Foram analisadas as escalas múltiplas de avaliação design do cardápio ( 3 itens), avaliação do restaurante (4 itens), sustentabilidade (4 itens) - e intenção de compra, com a ideia de comprar mais (4 itens), além das escalas simples de ponderação, como ida a restaurantes, preferência por ambientes que realizam ações sustentáveis, práticas individuais sustentáveis e a avaliação positiva ou negativa de que o restaurante pratica sustentabilidade. O resultado do teste foi significativo $(\mathrm{p}<0,01)$ para todas as escalas, considerando a amostra total e para cada grupo, rejeitando-se a hipótese nula de distribuição normal para todas as variáveis analisadas. Neste sentido, a utilização de técnicas paramétricas não é recomendada e optou-se, portanto, por técnicas não-paramétricas (Aaker; Kumar; Day, 2001; Malhotra, 2006; Corrar; Paulo; Dias Filho, 2007; Lattin; Carroll; Green, 2011).

Para o melhor entendimento do comportamento da escala, objetivando garantir a confiabilidade dos dados, realizou-se o cálculo do coeficiente alfa de Cronbach, que mede a consistência interna de escalas múltiplas (Malhotra, 2006; Hair et al., 2006). Nesta pesquisa, adotou-se apenas uma escala do tipo Liket de cinco pontos. Os testes apresentaram resultados acima de 0,6, ou seja, conforme Hair et al. (2006), valores acima deste resultado indicam confiabilidade satisfatória da consistência interna. Portanto, os resultados obtidos - design do cardápio $(=0,716)$, avaliação do restaurante $(=0,709)$, sustentabilidade $(=0,735)$ e intenção de compra $(=0,877)-$, apresentam-se satisfatórios.

Mesmo obtendo índices elevados com o alfa de Cronbach, que favorecem a afirmação de que há unidimensionalidade na escala utilizada, considerou-se necessário realizar uma análise fatorial para avaliar a estrutura interna das correlações entre as variáveis (Aranha; Zambaldi, 2008). A interpretação da magnitude do coeficiente de correlação não segue uma regra geral. Conforme Malhotra (2006), Hair et al. (2006) e Aranha \& Zambaldi (2008), amostras com mais de 100 unidades podem obter significância com cargas fatoriais a partir de, aproximadamente, 0,40. Aqui, optou-se por considerar como significativas as cargas a partir de 0,50. A Tabela 1 demonstra que as cargas fatoriais para esta escala apresentaram resultados acima do parâmetro estabelecido.

O cálculo do teste Kaiser-Meyer-Olkin (KMO) resultou em 0,741, demonstrando adequação das variáveis para aplicação do método. Segundo Malhotra (2006), valores entre 0,5 e 1 indicam que a análise fatorial é adequada. Para avaliar o risco de as variáveis não serem correlacionadas na população analisada, procedeu-se com o teste de esfericidade de Bartlett, obtendo-se o resultado do qui-quadrado de 637,49 com 105 graus de liberdade e nível de significância de $p<0,000$. Desta forma, com este resultado para a escala geral, estes resultados confirmaram a possibilidade da utilização do método de análise fatorial.

Analisando a Tabela 1, nota-se que há certa neutralidade nas respostas, de acordo com as médias apresentadas nas escalas para ambos os grupos de tratamento. As variáveis, em suas respectivas dimensões, foram mais bem avaliadas pelo grupo experimental. Com base nas médias dos grupos, observa-se que o restaurante, por meio da avaliação do cardápio, não foi tão bem avaliado. As médias um pouco mais elevadas estão na dimensão avaliação do restaurante, seguido da intenção de compra, isso para ambos os grupos.

As médias relacionadas ao design do cardápio foram as mais baixas em ambos os grupos. Cabe ressaltar que esta dimensão foi relevante para a pesquisa, porém não foi objetivo principal obter efeitos significativos neste fator. No entanto, é possível inferir que este aspecto é relevante para avaliação mais positiva de um restaurante (ver Strong, 2006). 
Tabela 1 - Cargas fatoriais, alfa de Cronbach e médias.

\begin{tabular}{|cccccc}
\hline Variáveis & $\begin{array}{c}\text { Carga } \\
\text { Fatorial }\end{array}$ & $\begin{array}{c}\text { Alfa de } \\
\text { Cronbach }\end{array}$ & $\begin{array}{c}\text { Média Geral } \\
\text { (desvio-padrão) }\end{array}$ & $\begin{array}{c}\text { Média (desvio-padrão) } \\
\text { GE }\end{array}$ & GC \\
\hline Design do cardápio & & 0,716 & & & \\
DES 1 & 0,671 & & $2,98(1,26)$ & $3,15(1,19)$ & $2,80(1,31)$ \\
DES 2 & 0,585 & & $2,65(1,12)$ & $2,79(1,12)$ & $2,50(1,12)$ \\
DES 3 & 0,518 & & $3,09(1,09)$ & $3,22(1,09)$ & $2,96(1,08)$ \\
\hline Avaliação do restaurante & & 0,709 & & & \\
AVAL 1 & 0,632 & & $3,40(1,00)$ & $3,60(0,95)$ & $3,20(1,02)$ \\
AVAL 2 & 0,575 & & $3,50(0,98)$ & $3,62(0,89)$ & $3,38(1,06)$ \\
AVAL 3 & 0,710 & & $3,84(0,82)$ & $3,96(0,72)$ & $3,72(0,91)$ \\
AVAL 4 & 0,512 & & $3,69(0,96)$ & $3,94(0,90)$ & $3,41(0,93)$ \\
Sustentabilidade & & 0,735 & & & \\
\hline SUST 1 & 0,610 & & $3,06(0,78)$ & $3,18(0,73)$ & $2,92(0,81)$ \\
SUST 2 & 0,514 & & $3,08(0,96)$ & $3,20(0,93)$ & $2,96(0,81)$ \\
SUST 3 & 0,615 & & $2,82(1,08)$ & $2,96(1,02)$ & $2,67(1,13)$ \\
SUST 4 & 0,677 & & $2,92(0,99)$ & $3,17(0,88)$ & $2,65(1,04)$ \\
\hline Intenção de compra & & 0,877 & & & \\
\hline INTCOMP 1 & 0,765 & & $3,24(1,20)$ & $3,41(1,15)$ & $3,07(1,24)$ \\
INTCOMP 2 & 0,792 & & $3,27(1,22)$ & $3,41(1,15)$ & $3,12(1,29)$ \\
INTCOMP 3 & 0,821 & & $3,27(1,20)$ & $3,44(1,12)$ & $3,09(1,26)$ \\
INTCOMP 4 & 0,560 & & $3,01(1,18)$ & $3,12(1,18)$ & $2,90(1,17)$ \\
\hline
\end{tabular}

Fonte: Dados da pesquisa (2011).

Observando apenas as médias dos grupos, nota-se uma ligeira diferença com resultados mais elevados para todas as dimensões do grupo de experimental. Entretanto, fez-se necessário realizar os testes de diferenças de médias para todas as variáveis com relação à variável independente deste estudo. Para a comparação das médias dos grupos, foi especificado o nível de significância $\mathrm{p}<0,05$, bastante comum em pesquisas na área de marketing (Szulanski; Jensen, 2004; Smith, 2006; Debruyne; Frambach; Moenaert, 2010).

Com base nos resultados dos testes de normalidade apresentados anteriormente, verificou-se que o teste mais adequado para comparar as médias seria o U de Mann-Whitney (Malhotra, 2006; Hair et al., 2006). Este é um teste estatístico para uma variável de medida em escala ordinal que compara a diferença de posição de suas populações, baseada em observações de duas amostras independentes. Calcula-se este teste como o número de vezes que um escore da amostra ou grupo 1 precede o escore do grupo 2, neste caso, os GE e GC, respectivamente. Para dados paramétricos com o mesmo tipo de amostras, o teste adequado seria o $t$ de dois grupos (Malhotra, 2006).

Com relação à dimensão avaliação do restaurante, é interessante notar que os consumidores homens tendem a acreditar mais do que as mulheres que o local parece ser organizado a partir da avaliação do cardápio $(\mathrm{p}<0,018)$. Talvez seja interessante analisar que aspectos relacionados à imagem do cardápio projetado tende a favorecer a avaliação masculina e desfavorecer a avaliação feminina.

Quanto aos testes de hipóteses, a primeira (H1) considerou que os consumidores terão atitudes mais positivas na avaliação de restaurantes que enfatizam práticas de sustentabilidade em seu cardápio. Já de início, pode-se considerar que as frequências de cada variável apresentam proximidade em ambos os grupos. Porém, ao analisar a variável Sust 4 'O restaurante está preocupado com questões de sustentabilidade', verifica-se que a diferença de média é expressiva e 
significante estatisticamente (tabela 2). Quanto às demais variáveis, os resultados do teste não favorecem base para concluir que as amostras pertencem a populações diferentes, conforme os resultados de significância estatística. Por outro lado, optou-se, em razão do resultado da variável Sust 4, pela aceitação da hipótese $\mathrm{H} 1$, já que os resultados de significância e valores de $z$ foram relevantes para tal. Ou seja, o argumento de sustentabilidade apresentado no cardápio produziu avaliações mais positivas do restaurante por parte dos consumidores.

Tabela 2 - Teste H1 - Avaliação mais positiva.

$\begin{array}{cccccc}\text { Variáveis } & \text { U Mann-Whitney } & \mathbf{Z} & \text { Sig. } & \text { GE } & \text { GC } \\ \text { SUST1 } & 1327,500 & -1,722 & 0,085 & & 2,92 \\ & 1367,000 & -1,384 & 0,166 & 3,18(0,73) & (0,81) \\ \text { SUST2 } & & & & 3,20(0,93) & 2,96 \\ & 1399,000 & -1,173 & 0,241 & & 2,81) \\ \text { SUST3 } & 1175,000 & -2,572 & 0,010 & 2,96(1,02) & (1,13) \\ & & & & 3,17(0,88) & (1,04) \\ \text { SUST4 } & & & & & \end{array}$

Fonte: Dados da pesquisa (2011).

Para testar a hipótese $\mathrm{H} 2$ de que os consumidores estão mais propensos a frequentar restaurantes que apresentam características de práticas sustentáveis em seu cardápio, considerou-se também os resultados do $U$ de Mann-Whitney para todas as variáveis da dimensão intenção de compra (ver tabela 3). Como na hipótese H1, os resultados demonstraram que os indivíduos tendem a neutralidade nas respostas em ambos os grupos; mesmo tendo médias superiores no grupo de experimento.

Assim, embora se tenham maiores médias com relação à importância dada à sustentabilidade, atitudes de recomendação, preferência e disposição a pagar mais por restaurantes que adotam práticas sustentáveis por parte do grupo experimental, verifica-se que, de acordo com a significância estatística obtida, os resultados do teste não fornecem base para concluir que as amostras pertencem a populações diferentes. Portanto, em razão dos resultados, rejeita-se a hipótese H2. Ou seja, os consumidores investigados não estão mais propensos a consumir alimentos de restaurantes que apresentam características de práticas sustentáveis em seu cardápio.

Tabela 3 - Teste H2 - Intenção de compra.

\begin{tabular}{cccccc}
\hline Variáveis & $\begin{array}{c}\text { U Mann-Whitney } \\
1333,000\end{array}$ & $-1,548$ & Sig. & GE & GC \\
& & & & 3,122 & \\
INTCOMP1 & 1388,000 & $-1,227$ & 0,220 & & 3,07 \\
& & & & $3,41(1,15)$ & $(1,24)$ \\
INTCOMP2 & 1347,500 & $-1,463$ & 0,143 & & $(1,29)$ \\
& & & & $3,44(1,12)$ & $(1,26)$ \\
INTCOMP3 & 1415,000 & $-1,066$ & 0,286 & & 2,90 \\
& & & & $3,12(1,18)$ & $(1,17)$ \\
\hline
\end{tabular}

Fonte: Dados da pesquisa (2011).

Os resultados da variável que indica se o consumidor está disposto a pagar mais por restaurantes que produzem alimentos por meio de práticas sustentáveis (Intcomp4) permitem concluir também que estes participantes não estão dispostos a pagar mais por restaurantes que produzem alimentos por meio de práticas sustentáveis. Com isso, percebe-se que os resultados dos testes de hipóteses, alinham-se com os achados de Manaktola \& Jauhari (2007) apenas com relação a não disposição em pagar mais por estes serviços.

Embora seja apenas uma das dimensões que compõem o conceito de sustentabilidade, alguns autores, como Okada \& Mais (2010), defendem que os consumidores estão dispostos a pagar 
mais por alternativas ambientalmente corretas. Além disso, para a amostra analisada, verificou-se também que as questões de sustentabilidade não interferem nas preferências dos consumidores.

\section{CONSIDERAÇÕES FINAIS}

A revisão da literatura indicou que o consenso sobre a influência de argumentos de sustentabilidade no comportamento dos consumidores ainda está longe de se estabelecer. Embora já se tenham algumas evidências empíricas de que estas questões podem influenciar positivamente nas atitudes das pessoas com relação à avaliação (Martin; Simintiras, 1995; Lee; Roberts; Bacon, 1997; Holden, 1999; Manaktola; Jauhari, 2007; Robinot; Giannelloni, 2010) e, em alguns casos, em suas intenções de compra (Okada; Mais, 2010), considera-se necessário desenvolver mais pesquisas neste campo, objetivando obter explicações mais precisas dessas relações.

Ao considerar aspectos de organização, qualidade e aconchego, a partir da avaliação do cardápio, observa-se que os resultados foram poucos significativos, ao contrário do que propõe Strong (2006). Entretanto, não se podem ignorar os indícios de influência positiva entre os consumidores homens para esta dimensão. Com isso, talvez seja interessante analisar que aspectos relacionados às características do cardápio projetado tendem a favorecer a avaliação masculina e desfavorecer a avaliação feminina. Isso leva a crer que outras metodologias podem ser utilizadas para entender melhor esse fenômeno sob outra perspectiva. Por outro lado, apesar de alguns autores indicarem que as mulheres tendem a ser mais sensíveis às questões de sustentabilidade (Klineberg; McKeever; Rothenbach, 1998), não se observou diferenças nas respostas deste item.

$\mathrm{O}$ argumento de sustentabilidade apresentado no cardápio produziu avaliações mais positivas do restaurante por parte dos consumidores, ou seja, o selo indicando que o estabelecimento pratica ações sustentáveis, apresentado como argumento de sustentabilidade, influenciou positivamente nas avaliações dos participantes. Por outro lado, verificou-se que os consumidores não estão propensos a frequentar mais estabelecimentos que apresentam características de práticas sustentáveis em seu cardápio. Além disso, os resultados ainda revelam que estes possíveis consumidores não estão dispostos a pagar mais por restaurantes que produzem alimentos por meio de práticas sustentáveis. Estes achados alinham-se com os de Manaktola \& Jauhari (2007), quando consideram que os indivíduos não estão dispostos a pagar mais por estes serviços. Porém, há controvérsias quando se trata de argumentos ecológicos, pois Okada \& Mais (2010) defendem que os consumidores estão dispostos a pagar mais por alternativas ambientalmente corretas. É possível supor que as questões de sustentabilidade também não interferem nas preferências dos consumidores.

A contribuição acadêmica deste estudo foi testar, em campo, conceitos que estão sendo criados para melhorar a imagem das empresas a partir do tema sustentabilidade, neste caso, o selo que indica práticas sustentáveis. O assunto está em evidência e muitas questões ainda precisam ser respondidas. Embora os resultados não tenham sido positivos para a maioria das análises causais, observa-se que há comportamentos favoráveis por parte das pessoas ao avaliar um estabelecimento que se utiliza de argumentos de sustentabilidade em instrumentos de comunicação. Ou seja, há indícios de que isso pode influenciar na avaliação mais positiva do estabelecimento, sendo esta, uma sugestão de aprofundamento em pesquisas futuras.

Por fim, pela sua delimitação, ou melhor, o fato de ter sido utilizado somente o cardápio como instrumento de comunicação, este estudo apresenta apenas um viés de uma problemática bastante ampla. Desse modo, sugere-se investigar as atitudes dos consumidores frente a estes selos em outras ferramentas de comunicação (por exemplo, websites), bem como em outros tipos de estabelecimentos de serviços.

\section{REFERÊNCIAS}

Aaker, D., Bagozzi, R. P. (1982). Attitudes toward public policy alternatives to reduce air pollution. Journal of Marketing, 1, 85-94. 
Aaker, D.A., Kumar, V., Day, G.S. (2001). Pesquisa de marketing. São Paulo: Atlas.

Andersson, T.D., Mossberg, L. (2004). The dinning experience: do restaurants satisfy customer needs? Food Service Technology, 4, 171-177.

Anderson, W. T. Jr., Cunningham, W. H. (1972). The socially conscious consumer. Journal of Marketing, 36, 23-32, July.

Aranha, F., Zambaldi, F. (2008). Análise fatorial em administração. São Paulo: Cengage Learning.

Awad, T. A. (2011) Environmental segmentation alternatives: buyers' profiles and implications. Journal of Islamic Marketing, 2(1), 55-73.

Baker, M. J., Parasuraman, A., Grewal, D., Voss, G. B. (2002). The influence of multiple store environment cues on perceived merchandise value and patronage intentions. Journal of Marketing, $66,120-141$.

Barbosa, M. L. A., Kovacs, M. H. (2004). Decodificando estratégias competitivas: Como criar uma imagem mental única e intangível de um serviço que é, em grande parte tangível e não particularmente único. In: Encontro De Marketing, 1, Porto Alegre. Anais...Porto Alegre: EMA.

Bayou, M. E., Bennett, L. B. (1992). Profitability analysis for table service restaurants. Cornell Hotel and Restaurant Administration Quartely. Global, 1, 49-55, abr.

Biloslavo, R., Trnavcevic, A. (2009). Web sites as tools of communication of a "green" company. Management Decision, 47(7), 1158-1173.

Bitner, M. J. (1992). Servicescapes: the impact of physical surroundings on customers and employees. Journal of Marketing, 56, 57-71.

Brooker, G. (1976). The self-actualizing socially conscious behavior. Journal of Consumer Research, 3, 107-112, September.

Castelli, G. (1999). Administração hoteleira. (6 ed.), Caxias do Sul: Educs.

Chen, T.B., Chai, L.T. (2010). Attitude towards the environment and green products: consumers' perspective. Management Science and Engineering, 4(2).

Churchill, G. (1999). Marketing research: methodological foundations. (7 ed.), Dryden.

Cohen, S.G., Ledford Jr., G.E. (1994). The effectiveness of self-managing teams: a quasiexperiment. Human Relations, 47(1), 13-43.

Cooper, D.R., Schindler, P.S. (2003). Métodos de pesquisa em administração. (7. ed.), Porto Alegre: Bookman.

Corrar, L.J., P., Edilson; Dias-Filho, J.M. (2007). Análise multivariada: para os cursos de administração, ciências contábeis e economia. São Paulo: Atlas. 
Debruyne, M., Frambach, R. T., Moenaert, R. (2010). Using the weapons you have: the role of resources and competitor orientation as enablers and inhibitors of competitive reaction to new products. The Journal of Product Innovation Management, 27(2), 179.

Dorf, M.E. (1992). Restaurants that works - Case study of the best in the industry, Whitney Library of Design.

Firat, D. (2009). Demographic and psychographic factors that affect environmentally conscious consumer behavior: a study at kocaeli university in turkey. Journal of American Academy of Business. Cambridge, 14(2), 323-329, Mar.

Gail, B. (1993). Menus that sell. Restaurant Hospitality, Cleveland, 77(5), 72-80.

Hair, J. F. Jr., Black, W. C., Babin, B. J., Anderson R. E., Tatham, R. L. (2006). Multivariate data analysis. (6 ed.) Prentice Hall: New Jersey.

Grant, A.M., Hofmann, D. A. (2011). Outsourcing inspiration: the performance effects of ideological messages from leaders and beneficiaries. Organizational Behavior and Human Decision Processes, 116(2), 173.

Green Restaurant Association. (2011). Recuperado em: 23/11/2011, de: <green.com/>.

Grimshaw, J., Campbell, M., Eccles, M., Steen, N. (2000). Experimental and quasi-experimental designs for evaluating guideline implementation strategies, 17, Suppl. 1, Oxford University Press, Printed in Great Britain.

Herberger, Roy A, Jr. (1975). The ecological product buying motive: a challenge for consumer education. The Journal of Consumer Affairs (pre-1986), 9(2), 187, Winter.

Holder, M., Berndt, A. (2011). The effect of changes in servicescape and service quality perceptions in a maternity unit. International Journal of Health Care Quality Assurance, 24(5), 389-405.

Houaiss, A., Villar, M. S. (2004). Dicionário Houaiss da Língua Portuguesa. Rio de Janeiro: Objetiva.

Hulland, J., Chow, Y.H., Lam, S. (1996). Use of causal models in marketing research: a review. International Journal of Research in Marketing, 13, 181-97.

Hume, S. et al. (1989). Consumers go green. Advertising Age, 25, 3-5, September.

Jones, P., Mifli, M. (2000). Menu development and analusis in UK restaurant chains. Tourism and Hospitality Research. UK, 15, 61-71, ago.

Kassarjian, H.H. (1971) Incorporating ecology into marketing strategy: the case of air pollution. Journal of Marketing, 35(3), 61-65, Jul.

Kinnear, Thomas C. et al. (1974) Ecologically concerned consumers: who are they? Journal of Marketing, 38(2), 20-24, April.

Kivela, J.J. (1997) Restaurant marketing: selection and segmentation in Hong Kong. International Journal of Contemporary Hospytality Management, 9(3). 
Klineberg, S.L., Mckeever, M., Rothenbach, B. (1998) Demographic predictors of environmental concern: It does make a difference how it's measured. Social Science Quarterly, 79(15), 734-753.

Kotler, P. (1973) Atmospherics as a marketing tool. Journal of Retailing. Greenwich, 49(4), 48-64.

Lages, N., Neto, A.V. (2002). Mensurando a consciência ecológica do consumidor: um estudo realizado na cidade de Porto Alegre. In: Encontro anual da Associação Nacional de Programas de Pós-Graduação em Administração, 26, Salvador. Anais... Rio de Janeiro: Anpad.

Laroche, M. et al. (2001). Targeting consumers who are willing to pay more for environmentally friend products. Journal of Consumer Marketing, 18(6).

Lattin, J., Carroll, D.J., Green, P.E. (2011). Análise de dados multivariados. São Paulo: Cengage Learning.

Lee, J. A., Holden, S. J. S. (1999). Understanding determinants of environmentally conscious behavior. Psychology \& Marketing, 16(5), Aug.

Leonard-Barton, D. (1981). Voluntarily simplicity lifestyles and energy consumption. Journal of Consumer Behavior, 8, 243-252, December.

Lewis, D. M. (1981) Determinants of reproductive success of the white-browed sparrow weaver, plocepasser mahali. New York: Springer Berlin/Heidelberg.

Lin, I.Y. (2004). Evaluating a servicescape: the effect of cognition and emotion. International Journal of Hospitality Management.

Malhotra, N.K. (2006). Pesquisa de marketing: uma orientação aplicada. (4. ed). Porto Alegre: Bookman.

Manaktola, K., Jauhari, V. (2007). Exploring consumer attitude and behavior towards green practices in the lodging industry in India. International Journal of Contemporary Hospitality Management, 19(5), 364-377.

Maricato, P. (2005). Marketing para bares e restaurantes. Rio de Janeiro: Senac Nacional.

Martin, B., Simintiras, A.C. (1995). The impact of green product lines on the environment: does what they know affect how they feel? Marketing Intelligence \& Planning, 13(4), 16.

Mayer, R. C., Davis, J. H. (1999). The effect of the performance appraisal system on trust for management: a field quasi-experiment. Neue Literatur in der Arbeitspychologie WS, 3(4).

Murphy, P. et al. (1978). Racial differences in consumer environmental concern. Journal of Consumer Affairs, 31(1), 53-69.

Okada, E. M., Mais, E. L. (2010). Framing the "Green" alternative for environmentally conscious consumers. Sustainability Accounting, Management and Policy Journal, 1(2), 222-234.

Ottman, J.A. (1994) Marketing verde. São Paulo: Makron Books. 
Pelaez, N. (2008). Processo de planejamento de cardápio: um estudo de caso num restaurante de Balneário Camboriú. Dissertação (mestrado), Mestrado em Turismo e Hotelaria, Univali, Balneário Camboriú.

Pereira, S. J. N., Ayrosa, E. A. T. (2004). Atitudes relativas a marcas e argumentos ecológicos: um estudo experimental. Revista Gestão.Org, 2(2), maio/ago.

Petroll, M.L.M., Damacena, C., Zanluchi. (2004). Apelos sexuais em propagandas no contexto brasileiro: análise da atitude em relação à propaganda, marca, ética e intenção de compra. In: Encontro Anual da Associação dos Programas de Pós-Graduação em Administração. Anais....

Roberts, J.A. (1996). Green consumers in the 1990s: profile and implications for advertising. Journal of Business Research, 36(3), 217-231.

Roberts, J.A., Bacon, D.R. (1997). Exploring the subtle relationships between environmental concern and ecologically consumer behavior. Journal of Business Research, 40(1), 79-89.

Robinot, E., Giannelloni, J.-L. (2010). Do hotels' "green" attributes contribute to customer satisfaction? The Journal of Services Marketing, 24(2), 157-169.

Rowlands, et al. (2003). Consumers and green electricity: profiling potential purchasers. Business Strategy and the Environment, 36-48.

Samdahl, D.M., Robertson, R. (1989). Social determinant of environmental concern: specification and test of the model. Environment and Behavior, 21(1). 5-81.

Sampieri, R.H., Collado, C.F., Lucio, P.B. (2006). Metodologia de Pesquisa. (3.ed.) São Paulo: McGraw-Hill.

Serpa, D.A., Avila, M.G. (2000). Efeito framing e influência da experiência gerencial em marketing em decisões de compra: um teste experimental. In: Encontro Anual da Associação dos Programas de Pós-Graduação em Administração, Florianópolis. Anais... Florianópolis.

Schwepker, Jr., C. H., Cornwell, T. B. (1991). An examination of ecologically concerned consumers and their intentions to purchase ecologically package products. Journal of Public Policy and Marketing, 10, 77-101.

Shadish, W. R., Cook, T. D., Campbell, D. T. (2002). Experimental and quasi-experimental designs for generalized causal inference. Houghton Mifflin Company, Boston, New York.

Smith, C.D. (2001). Environmentalism, feminism and gender. Sociological Inquiry, 71(3), 314-334, Summer.

Smith, A. (2006). Assessing the contribution of flagship projects to city image change: a quasiexperimental technique. The International Journal of Tourism Research, Chichester, 8(6), 391, nov./dec.

Soriano, D. R. (2002). Customers expectation factors in restaurants: the situation in Spain. The International Journal of Quality \& Reliability Management. Valencia, 19(8/9), 1055-1067.

Strong, A. (2006). Nice menu you got there. Restaurant Business, New York, 105(4), 25-29, abr. 
Szulanski, G., Jensen, R. J. (2004). Overcoming stickiness: an empirical investigation of the role of the template in the replication of organizational routines. Managerial and Decision Economics, Chichester, 25(6-7), 347.

Szybilo, G. J., Jacoby, J. (1974). Intrinsic versus extrinsic cues as determinants of perceived product quality. Journal of Applied Psychology, 59,74-78.

Tucker, L. R, Jr. (1980). Identifying the environmentally responsible consumer: the role of internal external control of reinforcements. The Journal of Consumer Affairs, Winter (pre-1986), 14(2), 326.

Walker, J. R., Lundberg, D. E. (2003). O restaurante: conceito e operação. Porto Alegre: Bookman.

Winer, R. S. (1999). Experimentation in the 21st century: the importance of external validity. Academy of Marketing Science. Journal, 27(3), 349, Summer.

Velter, A. N., Battistella, L. F., Grohmann, M. Z., Castro, A. E., Costa, V. F., Hermann, R. E. (2009). Atitudes dos consumidores a partir da teoria das pistas e consciência ambiental: contribuições ao estudo do Green Marketing. Revista de Administração UFSM, Santa Maria, 2(3), 399-416, set./dez.

Zimmer, M. R. et al. (1994). Green issues: dimensions of environmental concern. Journal of Business Research, 30(1), 63-74.

Recebido em: 11/10/2011

Publicado em: 21/12/2012 